\title{
Improving effects of chitosan nanofiber scaffolds on osteoblast proliferation and maturation
}

This article was published in the following Dove Press journal:

International Journal of Nanomedicine

9 September 2014

Number of times this article has been viewed

\author{
Ming-Hua $\mathrm{Ho}^{1,2}$ \\ Mei-Hsiu Liao ${ }^{3}$ \\ Yi-Ling Lin ${ }^{2}$ \\ Chien-Hao Lai ${ }^{3}$ \\ Pei-I Lin ${ }^{3}$ \\ Ruei-Ming Chen ${ }^{2-4}$ \\ 'Department of Chemical Engineering, \\ National Taiwan University of Science \\ and Technology, Taipei, Taiwan; ${ }^{2} \mathrm{Cell}$ \\ Physiology and Molecular Image \\ Research Center and Department of \\ Anesthesiology, Wan Fang Hospital, \\ ${ }^{3}$ Graduate Institute of Medical \\ Sciences, Taipei Medical University, \\ Taipei, Taiwan; ${ }^{4}$ Anesthetics and \\ Toxicology Research Center, Taipei \\ Medical University Hospital, Taipei, \\ Taiwan
}

\begin{abstract}
Osteoblast maturation plays a key role in regulating osteogenesis. Electrospun nanofibrous products were reported to possess a high surface area and porosity. In this study, we developed chitosan nanofibers and examined the effects of nanofibrous scaffolds on osteoblast maturation and the possible mechanisms. Macro- and micro observations of the chitosan nanofibers revealed that these nanoproducts had a flat surface and well-distributed fibers with nanoscale diameters. Mouse osteoblasts were able to attach onto the chitosan nanofiber scaffolds, and the scaffolds degraded in a time-dependent manner. Analysis by scanning electron microscopy further showed mouse osteoblasts adhered onto the scaffolds along the nanofibers, and cell-cell communication was also detected. Mouse osteoblasts grew much better on chitosan nanofiber scaffolds than on chitosan films. In addition, human osteoblasts were able to adhere and grow on the chitosan nanofiber scaffolds. Interestingly, culturing human osteoblasts on chitosan nanofiber scaffolds time-dependently increased DNA replication and cell proliferation. In parallel, administration of human osteoblasts onto chitosan nanofibers significantly induced osteopontin, osteocalcin, and alkaline phosphatase (ALP) messenger (m)RNA expression. As to the mechanism, chitosan nanofibers triggered runt-related transcription factor 2 mRNA and protein syntheses. Consequently, results of ALP-, alizarin red-, and von Kossa-staining analyses showed that chitosan nanofibers improved osteoblast mineralization. Taken together, results of this study demonstrate that chitosan nanofibers can stimulate osteoblast proliferation and maturation via runt-related transcription factor 2-mediated regulation of osteoblast-associated osteopontin, osteocalcin, and ALP gene expression.
\end{abstract}

Keywords: chitosan nanofibers, osteoblast-associated gene expression, osteoblast maturation, Runx2

\section{Introduction}

Bone contributes to tissue protection, physical movement, and skeletal support. In the clinic, bone diseases such as bone defects and fractures in patients may lead to disability or even death. Anatomically, the bone structure is maintained by bone remodeling, which is a balanced and dynamic process of bone formation and resorption. ${ }^{1}$ Osteoblasts, which differentiate from stromal stem cells, were reported to play a central role in mediating bone formation. ${ }^{2,3}$ Osteogenesis is the process of bone formation and consists of various consecutive stages, including osteoprogenitor proliferation, matrix maturation, and osteoblast mineralization. ${ }^{4,5}$ In the beginning of bone turnover or fracture healing, stem cells and primitive osteoprogenitors proliferate and subsequently replace old and damaged osteoblasts. These precursor cells successively undergo sequential differentiation and finally achieve osteoblast maturation and bone nodule formation. ${ }^{6}$ However, multiple factors participate in controlling osteoblast maturation. Thus, to develop innovative biomaterials for therapy of bone diseases, it is important
Correspondence: Ruei-Ming Chen Graduate Institute of Medical Sciences, College of Medicine, Taipei Medical University, $250 \mathrm{Wu}$-Xing St, Taipei, II0, Taiwan

Tel +88622736 I $66 \mid$ ext 3222

Fax +88628662 III9

Email rmchen@tmu.edu.tw 
to evaluate the mechanisms of how biomaterials regulate osteoblast development.

A complicated network of molecular events is involved in regulating bone development., ${ }^{7,8}$ These progressive events are tightly regulated by sequential induction of osteoblast differentiation-related genes and are closely associated with the development of bone cell function. Alkaline phosphatase (ALP), osteopontin (OPN), and osteocalcin (OCN) are early osteoblast markers that participate in controlling osteoblast function and bone extracellular matrix (ECM) mineralization. ${ }^{9,10}$ Upregulation of OPN, ALP, and OCN is directly correlated with osteoblast differentiation. ${ }^{4,9}$ Also, OPN can function as a bridge between the bone and blood by adjusting the proliferation of hematopoietic stem cells. ${ }^{11}$ Regulation of these osteoblast-associated gene expression typically occurs at a transcriptional level. Runt-related transcription factor 2 (Runx2), an osteogenic master transcription factor, was reported to regulate osteoblast differentiation and maturation. ${ }^{12,13}$ Previous studies showed that activation of Runx2 induces ALP, OCN, and OPN messenger (m)RNA expression and concurrently promotes osteoblast differentiation. ${ }^{14,15}$ Thus, Runx2-involved regulation of osteoblast-associated gene expression makes a proportionally major contribution to regulating osteoblast proliferation and maturation.

Bone tissue engineering utilizes both engineering and life sciences to either maintain existing bone tissue structures or enable bone tissue growth. ${ }^{16}$ Biomaterials used for bone engineering may be comprised, wholly or in part, of a living structure or biomedical device that performs, augments, or replaces a natural function. To fulfill the functions of a biomaterial for bone tissue, the material should meet certain requirements of absorption kinetics, and have interconnected micropores and optimal porosity. ${ }^{17}$ However, one major reason for orthopedic implant failure is the lack of sufficient integration of the implanted material into the juxtaposed bone. ${ }^{18}$ Chitosan nanofibers possess a high surface area and porosity, and their use for fabricating biocompatible and biomimetic nanostructure scaffolds in tissue engineering has been investigated. ${ }^{19}$ Previous studies showed that chitosan nanofibers/polycaprolactone scaffolds provided good bipolar elongation of neurons to nanofibrous substrates, such that these nanofibrous scaffolds can maintain a cell's morphology and stimulate cell proliferation..$^{20,21}$ Chen et al further reported that chitosan nanofibers can be applied as a biomimetic ECM for endothelial and smooth muscle cells. ${ }^{22}$ In cartilage engineering, chitosan nanofibers can improve the resistance of porous scaffolds to compressive loading, which can provide more structural protection to seeded mesenchymal stem cells when the construct is implanted into a lesion. ${ }^{23}$ In addition, Liu et al demonstrated the effects of nanofibrous hydroxyapatite/chitosan scaffolds on promoting bone marrow mesenchymal stem cell activities. ${ }^{24}$ In this study, we developed chitosan nanofibrous products and examined their effects on osteoblast maturation as well as the possible action mechanisms.

\section{Materials and methods Materials}

Chitosan with a deacetylation degree of $85 \%$, trifluoroacetic acid, 3-(4,5-cimethylthiazol-2-yl)-2,5-diphenyl tetrazolium bromide, bromodeoxyuridine (BrdU), and crystal violet were purchased from Sigma-Aldrich Co. (St Louis, MO, USA). The molecular weight of chitosan used in this study was $210 \mathrm{kDa}$. Dichloromethane was purchased from Tedia (Fairfield, OH, USA). $\alpha$-Minimum Essential Medium and Dulbecco's Modified Eagle's Medium were obtained from Thermo Fisher Scientific (Waltham, MA, USA). To evaluate the effects of chitosan on osteoblasts, three different types of osteoblast-like cell lines were used in this study: UMR-106 cells, originally isolated from a rat osteosarcoma; MC3T3E1 cells, osteoblast precursor cells derived from mouse calvarias; and MG63 cells, derived from a human osteosarcoma.

\section{Electrospinning of chitosan}

Chitosan nanofiber scaffolds were prepared following a previously described method. ${ }^{25}$ Briefly, the electrospinning setup used in this study consisted of three major components: a power supply using direct current that could generate a voltage of up to $30 \mathrm{kV}$; a $3 \mathrm{~mL}$ syringe with a metallic needle with a $0.65 \mathrm{mM}$ inner diameter that could control the flow rate of a scientific pump (model 780/00; KD Scientific, Holliston, MA, USA); and a collector made from aluminum foil for fiber collection. Chitosan was dissolved in trifluoroacetic acid/dichloromethane $(70 / 30 \mathrm{vol} \%)$ at a concentration of $50 \mathrm{mg} / \mathrm{mL}$, and the electrospinning solutions were stirred for 24 hours into well-mixed homogeneous solutions. The tip-to-collector distance was $12 \mathrm{~cm}$, and the applied voltage was $17 \mathrm{kV}$. The rate of chitosan feeding in the electrospinning was $0.2 \mathrm{~mL} / \mathrm{h}$. Before being applied to the following physical tests and biomedical assays, the chitosan nanofibers were neutralized by reaction with saturated sodium carbonate at $25^{\circ} \mathrm{C}$ for 5 hours.

\section{Scanning electron microscopy (SEM)}

The surface morphology of the chitosan nanofiber scaffolds was observed and photographed using SEM (JSM-6390LV; JEOL, Tokyo, Japan) at an accelerating voltage of $15 \mathrm{kV}$ after 
the surface was coated with gold. To prepare samples of cellcontaining scaffolds for the SEM analysis, osteoblasts were fixed and dehydrated before gold coating. The chitosan nanofiber scaffolds with osteoblasts were fixed with a $1 \%$ glutaraldehyde solution and dehydrated in a series of ethanol aqueous solutions with successively increasing ethanol concentration of $30 \%-99.99 \%$. Critical-point drying with supercritical $\mathrm{CO}_{2}$ was then performed to prevent cell deformation. Surfaces of the scaffolds were coated with gold and scanned using SEM.

\section{Cell cultures and drug treatment}

UMR-106 cells were cultured in $\alpha$-Minimum Essential Medium, and MC3T3-E1 cells and MG63 cells were seeded in Dulbecco's Modified Eagle's Medium. Both media were supplemented with $10 \%$ fetal bovine serum, L-glutamine, penicillin $(100 \mathrm{IU} / \mathrm{mL})$, and streptomycin $(100 \mu \mathrm{g} / \mathrm{mL})$ in $75 \mathrm{~cm}^{2}$ flasks at $37^{\circ} \mathrm{C}$ in a humidified atmosphere of $5 \% \mathrm{CO}_{2}$. When osteoblasts were confluent, cells were subcultured into petri dishes coated with chitosan films or chitosan nanofiber scaffolds for various time intervals for different assays.

\section{Biocompatibility of chitosan nanofiber scaffolds}

Osteoblasts were seeded in $6 \mathrm{~cm}$ tissue culture dishes coated with chitosan nanofiber scaffolds. After culturing for different time intervals, the scaffolds were observed and photographed using a light microscope (Eclipse 50i; Nikon Corporation, Tokyo, Japan).

\section{Mitochondrial complex I enzyme activity assay}

Activity of the mitochondrial complex I enzyme was assayed to evaluate the effects of chitosan films and chitosan nanofiber scaffolds on osteoblast growth according to a previously described method. ${ }^{26}$ Osteoblasts $\left(2 \times 10^{4}\right.$ cells/well $)$ were seeded in 96-well tissue culture plates coated with chitosan films or chitosan nanofiber scaffolds overnight. After treatment, osteoblasts were cultured with new medium containing 3-(4,5-cimethylthiazol-2-yl)-2,5-diphenyl tetrazolium bromide at $0.5 \mathrm{mg} / \mathrm{mL}$ for a further 3 hours. The blue formazan products in the osteoblasts were dissolved in dimethyl sulfoxide and spectrophotometrically measured at a wavelength of $550 \mathrm{nM}$.

\section{Crystal violet staining}

A crystal violet assay was carried out and quantified according to a previously described method..$^{27}$ Briefly, osteoblasts were cultured in petri dishes coated with chitosan nanofiber scaffolds.
After culturing for different time intervals, the dishes were rinsed with phosphate-buffered saline (PBS; 0.14 M NaCl, $2.6 \mathrm{mM} \mathrm{KCl}, 8 \mathrm{mM} \mathrm{Na}_{2} \mathrm{HPO}_{4}$, and $\left.1.5 \mathrm{mM} \mathrm{KH}_{2} \mathrm{PO}_{4}\right)$. The cell colonies were stained with a $0.2 \%$ crystal violet solution in $10 \%$ ethanol for 10 minutes. Excess stain was removed by washing repeatedly with PBS. Stained cells were observed and photographed using light microscopy (Nikon Corporation). The crystal violet that had stained cells from each dish was dissolved in $1 \mathrm{~mL}$ of $10 \%$ acetic acid. The solution was spectrophotometrically measured at a wavelength of $590 \mathrm{nM}$.

\section{Cell proliferation assay}

The effect of chitosan nanofiber scaffolds on cell proliferation was analyzed by measuring the incorporation of BrdU into genomic DNA..$^{28}$ Osteoblasts were seeded at $3 \times 10^{3}$ cells/well in 96-well microtiter plates. After culturing for different time intervals, $10 \mathrm{mM} \mathrm{BrdU}$ was added to the culture medium for incorporation into the DNA of replicating cells. After 2 hours of incubation, cells were fixed in 4\% paraformaldehyde. BrdU incorporation was determined by a cell-proliferation enzyme-linked immunosorbent assay BrdU kit (Hoffman-La Roche Ltd., Basel, Switzerland).

\section{Reverse transcription (RT)-polymerase chain reaction (PCR) assay}

mRNA from macrophages exposed to drugs was prepared for RT-PCR analyses of OPN, OCN, ALP, Runx2, and $\beta$-actin mRNAs according to a previously described method. ${ }^{29}$ Oligonucleotides for the PCR analyses of these mRNAs were designed and synthesized by Takara Bio Inc. (Otsu, Japan). The oligonucleotide sequences of the upstream and downstream primers for these mRNA analyses were,

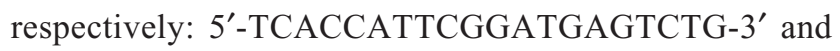
5' - ACTTGTGGCTCTGATGTTCC-3' for OPN; ${ }^{30} 5^{\prime}$-ATGAGGACCCTCTCTCTGCTC-3' and 5'-GTGGTGCCATAATGCGCTTG-3' for OCN $;^{26} 5^{\prime}$-CCAACTCTTTTGTGCCAGAGA-3' and 5'-GGCTACATTGGTGTTGAGCTTTT-3' for ALP; ${ }^{21}$ 5'-GACA GAA GCT T GA T GACTCTAAACC-3' and 5'-CTGTAATCTGACTCTGTCCTTGTG-3' for Runx $2 ;^{13}$ and $5^{\prime}$-GTGGGCCGCTCTAGGCACCAA-3' and $5^{\prime}$-CTCTTTGATGTCACGCACGATTTC- $3^{\prime}$ for rat $\beta$-actin. ${ }^{13}$ The PCR products were loaded onto a $1.8 \%$ agarose gel containing $0.1 \mu \mathrm{g} / \mathrm{mL}$ ethidium bromide, and were electrophoretically separated. DNA bands were visualized and photographed under ultraviolet-light exposure. The intensities of the DNA bands in the agarose gel were quantified with the aid of the UVIDOCMW vers. 99.03 digital imaging system (UVtec, Cambridge, UK). 


\section{Immunodetection of Runx2 and $\beta$-actin proteins}

After treatment, cell lysates were prepared in ice-cold radioimmunoprecipitation assay buffer $(25 \mathrm{mM}$ Tris- $\mathrm{HCl}$ [pH 7.2], 0.1\% sodium dodecyl sulfate, $1 \%$ Triton X-100, $1 \%$ sodium deoxycholate, $0.15 \mathrm{M} \mathrm{NaCl}$, and $1 \mathrm{mM}$ ethylenediaminetetraacetic acid [EDTA]) as described previously. ${ }^{31}$ To avoid protein degradation, a mixture of proteinase inhibitors, including $1 \mathrm{mM}$ phenyl methyl sulfonyl fluoride, $1 \mathrm{mM}$ sodium orthovanadate, and $5 \mathrm{mg} / \mathrm{mL}$ leupeptin, was added to the radioimmunoprecipitation assay buffer. Protein concentrations were quantified by a bicinchoninic acid protein assay kit (Thermo Fisher Scientific). Cytosolic proteins (100 mg/ well) were subjected to sodium dodecyl sulfate-polyacrylamide gel electrophoresis and transferred to nitrocellulose membranes. These membranes were blocked with 5\% non-fat milk at $37^{\circ} \mathrm{C}$ for 1 hour. Runx 2 were immunodetected using a mouse monoclonal antibody against Runx2 (Santa Cruz Biotechnology Inc., Dallas, TX, USA). $\beta$-actin was detected using a mouse monoclonal antibody (Sigma-Aldrich Co.) as the internal control. These protein bands were quantified using a digital imaging system (UVtec).

\section{Assays of osteoblast mineralization}

Osteoblast maturation was determined by evaluating cell mineralization using the ALP-, von Kossa-, and alizarin red S dye-staining protocols. ${ }^{26,32}$ Osteoblasts were seeded in $6 \mathrm{~cm}$ tissue culture dishes coated with chitosan nanofiber scaffolds, and were treated with a combination of $10 \mathrm{nM}$ dexamethasone, $100 \mu \mathrm{g} / \mathrm{mL}$ ascorbic acid, and $10 \mathrm{mM} \beta$-glycerophosphate for 21 days. After drug treatment, osteoblasts were washed with ice-cold PBS and then fixed in ice-cold 10\% formalin for 20 minutes. For the ALP staining protocol, cells were stained at $37^{\circ} \mathrm{C}$ in a solution containing naphthol AS-MX phosphate disodium salt, fast red salt (Sigma-Aldrich Co.) and $N, N$-dimethyl formamide for 30 minutes. Cells were washed with PBS and photographed using phase-contrast microscopy. The ALP activity was assessed as red stains indicating the products of enzyme activity. For the von Kossa protocol, mineralized matrix was detected by treating fixed cells with $5 \%$ silver nitrate for 30 minutes, followed by subsequent washes with 5\% sodium carbonate in $10 \%$ formalin for 1 minute and 5\% sodium thiosulfate for 5 minutes. The reaction was stopped by washing cells twice with deionized water. For the alizarin red S dye protocol, fixed osteoblasts were thoroughly rinsed and then incubated in 1\% Alcian Blue pH 2.5 (Thermo Fisher Scientific) for 12 hours. Sections were then incubated in alizarin red S (Thermo Fisher Scientific) for
8 minutes, dehydrated briefly in xylene, and covered with a coverslip in Permount (Thermo Fisher Scientific). Mineralized nodules were visualized and counted under an inverted microscope. Each experiment was performed in duplicate wells and repeated three times.

\section{Statistical analyses}

The statistical significance of differences between the control and drug-treated groups was evaluated using Student's $t$-test, and differences were considered statistically significant at $P$-values of $<0.05$. Differences between drug-treated groups were considered significant when the $P$-value of Duncan's multiple-range test was $<0.05$. Statistical analyses between groups over time were carried out by a two-way analysis of variance.

\section{Results \\ Macro- and microstructures of the chitosan nanofiber scaffolds}

Chitosan nanofiber scaffolds were prepared using an electrospinning system and their physical properties were analyzed (Figure 1). The thickness, measured from SEM images, and the Young's modulus of the chitosan nanofiber were $225 \pm 21 \mu \mathrm{m}$ and $116 \pm 32 \mathrm{MPa}$, respectively. Also, the swelling degree of the chitosan nanofibrous substrate was around $700 \%$. Large-magnification observations showed that the surface structure of the chitosan nanofiber scaffolds was flat (Figure 1A). When examined under light microscopy, the electrospun fibers in the chitosan scaffolds were visible (Figure 1B). The high porosity of the fibrous scaffold was also revealed. The scaffolds were further scanned using SEM, and the image showed that the diameters of these electrospun fibers were at the nano level (Figure 1C).

\section{Mouse osteoblasts adhered to chitosan nanofiber scaffolds and degraded the materials}

Analysis of crystal violet staining revealed that mouse osteoblasts had attached onto the chitosan films (Figure 2Aa). After chitosan was modified into nanofibers, mouse osteoblasts also adhered onto the nanoscale scaffolds (Figure 2Ab). Adhesion of mouse osteoblasts onto chitosan nanofiber scaffolds was further verified using SEM (Figure 2B). Our results indicated that mouse osteoblasts could tightly attach to scaffolds along the nanofibers. Also, cell-cell communication among mouse osteoblasts was also monitored (Figure 2B). Analysis using light microscopy demonstrated that, after culturing for 1, 3, and 5 days, mouse osteoblasts had gradually degraded the chitosan nanofibers (Figure 2C). 

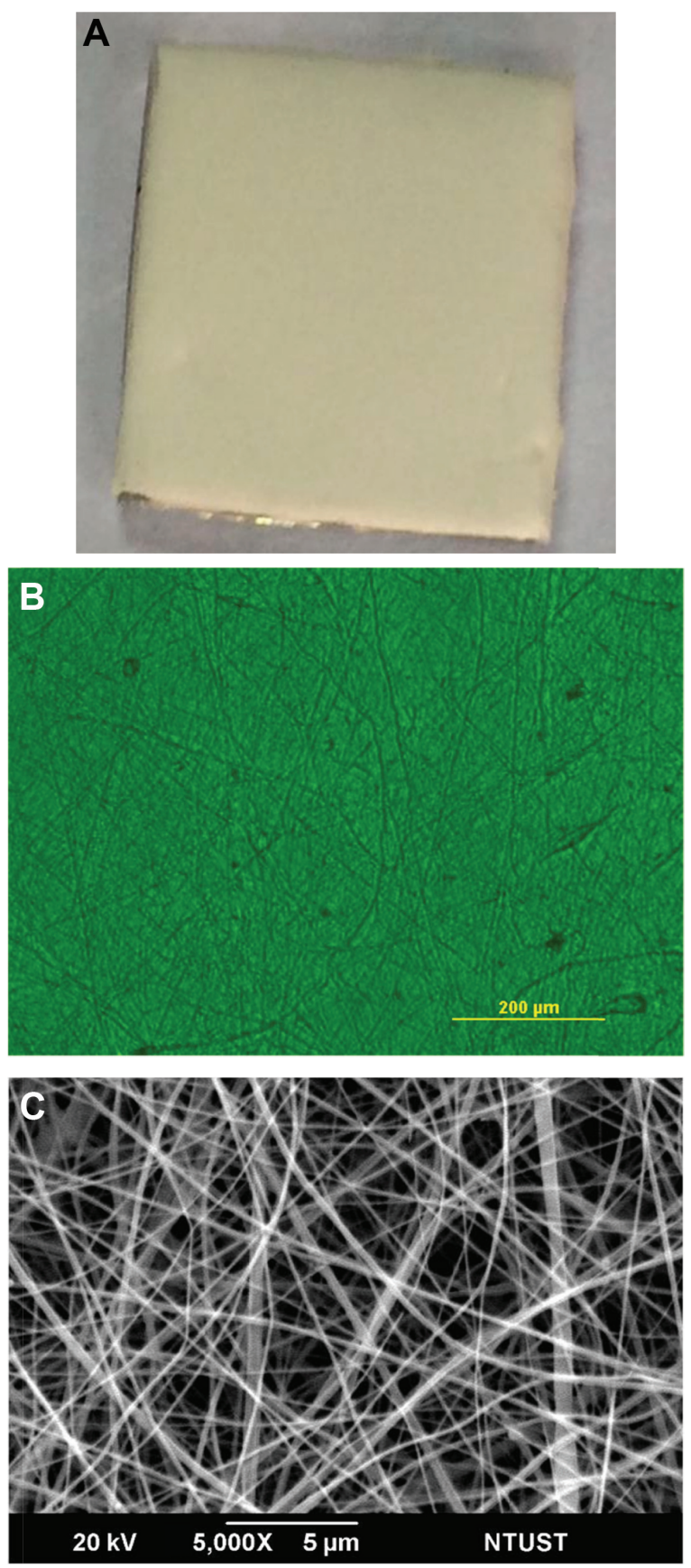

Figure I Macro- and microstructures of chitosan nanofibers

Notes: Chitosan nanofiber scaffolds were prepared using an electrospinning setup. The macro- (A) and microstructures (B) of chitosan nanofibers were observed and photographed using a camera and light microscopy, respectively. The surface morphology of chitosan nanofiber scaffolds was further scanned and photographed using scanning electron microscopy (C).

Abbreviation: NTUST, National Taiwan University of Science and Technology.

\section{Mouse osteoblasts grew on the chitosan nanofiber scaffolds}

After verifying the adhesion of mouse osteoblasts onto chitosan nanofibers, growth of these osteoblasts on the scaffolds was assayed (Figure 3). Analysis of mitochondrial complex I enzyme activity showed that, after seeding mouse osteosarcoma
UMR-106 cells onto chitosan films and chitosan nanofiber scaffolds for 3 and 5 days, cell growth increased by $45 \%$ and $70 \%$, respectively (Figure 3A). Five days after culturing on chitosan films and chitosan nanofiber scaffolds, growth of mouse osteoblasts was respectively augmented by 2.4 - and 3.9-fold. Use of chitosan nanofibers resulted in a significant $63 \%$ increase in cell growth (Figure 3A) compared to chitosan films.

Effects of the chitosan nanofiber scaffolds on cell growth were further evaluated using a different type of mouse osteoblast - MC3T3-E1 cells - derived from mouse calvaria, as the experimental model (Figure 3B). Investigation by crystal violet staining revealed that 1,3 , and 5 days after culturing onto chitosan nanofiber scaffolds, MC3T3-E1 cells had obviously grown in a time-dependent manner. Stained cells were dissolved, and the signals were quantified and statistically analyzed (Figure 3C). Three and 5 days after seeding MC3T3E1 cells onto chitosan nanofiber scaffolds, cell growth had significantly increased by $74 \%$ and 2.1 -fold, respectively (Figure 3C).

\section{Human osteoblasts grew and proliferated on chitosan nanofiber scaffolds}

Effects of the chitosan nanofiber scaffolds on attracting and stimulating cell adhesion and cell growth were further explored using human osteoblast MG63 cells as the experimental model (Figure 4). Human osteoblasts adhered onto the chitosan nanofiber scaffolds (Figure 4A). One, 3, and 5 days after culturing human osteoblasts on nanofibrous scaffolds, cell growth was time-dependently enhanced. Cell densities were quantified and statistically analyzed (Figure 4B). Use of human osteoblasts on chitosan nanofiber scaffolds for 1, 3, and 5 days caused significant 2.1-, 2.7-, and 3.7-fold increases in cell growth, respectively. A BrdU incorporation assay was further carried out (Figure 4C). When human osteoblasts were cultured on chitosan nanofiber scaffolds, the rates of DNA replication were augmented by $35 \%, 66 \%$, and $132 \%$, respectively (Figure 4C).

\section{Chitosan nanofiber scaffolds induced osteoblast differentiation-related gene expression}

RNA analyses were conducted to determine the effects of chitosan nanofiber scaffolds on regulation of osteoblastassociated OPN, OCN, and ALP gene expression (Figure 5). Exposure of human osteoblasts to chitosan nanofibers for 3 and 5 days increased OPN mRNA expression (Figure 5A, OPN panel, lanes 3 and 4). After 5 days of culture, OCN mRNA was induced by chitosan nanofibers (Figure 5A, OCN 
A

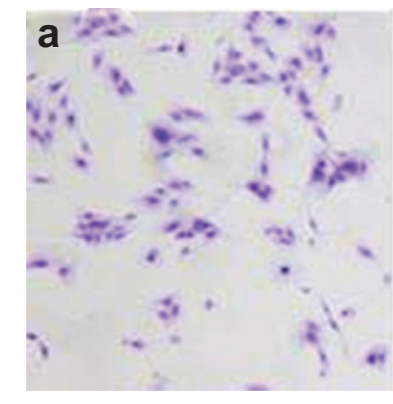

C

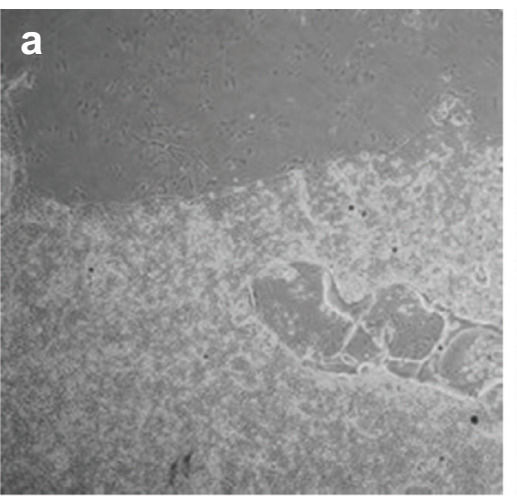

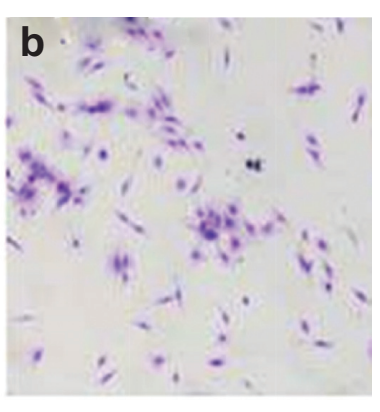
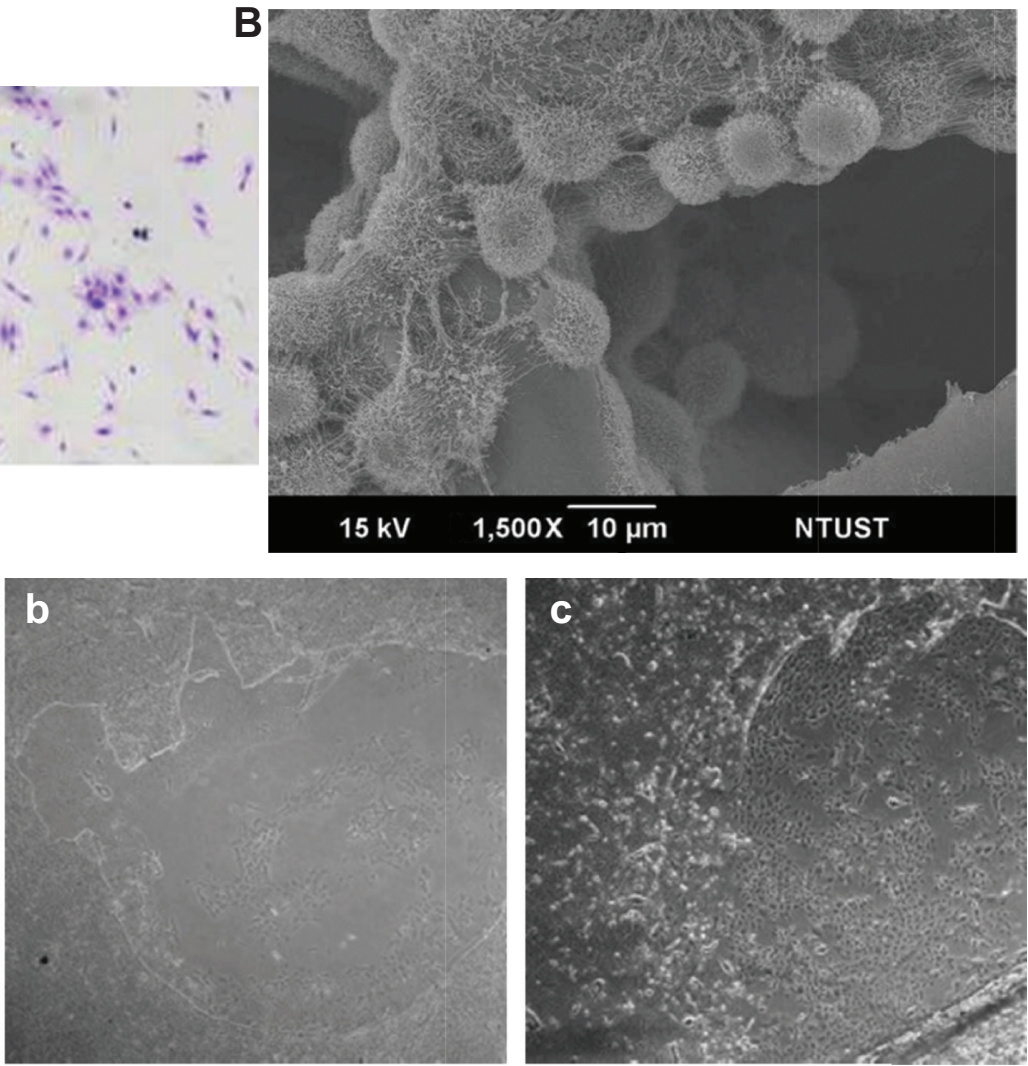

Figure 2 Osteoblast adhesion and biocompatibility of chitosan nanofibers.

Notes: Mouse osteoblasts were seeded overnight in $6 \mathrm{~cm}$ tissue culture plates coated with chitosan nanofiber scaffolds. Cell adhesion was assayed using a crystal violet staining protocol (A): (a) CS film; (b) CS nanofibers. Also, adhesion of mouse osteoblasts onto chitosan nanofiber scaffolds was further analyzed using scanning electron microscopy (B). (C) After culturing mouse osteoblasts on chitosan nanofiber scaffolds for (a) I, (b) 3, and (c) 5 days, the biocompatibility of the electrospun scaffolds was observed and photographed using light microscopy. 40× magnification.

Abbreviations: CS, chitosan; NTUST, National Taiwan University of Science and Technology.

panel, lane 4). In parallel, levels of ALP mRNA in human osteoblasts were augmented following exposure to chitosan nanofiber scaffolds for 5 days (Figure 5A, ALP panel, lane 4). Amounts of $\beta$-actin mRNA were determined as the internal control (Figure 5A, $\beta$-actin panel). The DNA bands were quantified and statistically analyzed (Figure 5B-D). Use of human osteoblasts with chitosan nanofiber scaffolds for 3 and 5 days, respectively, caused significant $81 \%$ and 2.3-fold increases in OPN mRNA expression (Figure 5B). In comparison, after seeding human osteoblasts onto chitosan nanofiber scaffolds for 5 days, the amounts of OCN and ALP mRNA were significantly augmented by $64 \%$ and $66 \%$, respectively (Figure 5C and D).

\section{Chitosan nanofiber scaffolds stimulated transcription factor Runx2 mRNA and protein expression}

To evaluate the mechanism of chitosan nanofiber-induced osteoblast-associated gene expression, levels of Runx2 mRNA and protein were further assessed (Figure 6).
Application of human osteoblasts onto chitosan nanofibers for 1 day increased the level of Runx 2 mRNA (Figure 6A, top panel, lane 2). Three, 5, and 7 days after exposure to chitosan nanofibers, Runx 2 mRNA expression was induced in a time-dependent manner (Figure 6A, lanes 3-5). $\beta$-actin mRNA was analyzed as the internal control (Figure 6A, bottom panel). The DNA bands were quantified and statistically analyzed (Figure 6B). After 1, 3, 5, and 7 days of culturing human osteoblasts on chitosan nanofiber scaffolds, amounts of Runx 2 mRNA were significantly induced by $67 \%, 88 \%$, $92 \%$, and $163 \%$, respectively (Figure 6B). Administration of human osteoblasts with chitosan nanofibers for 1, 3, 5, and 7 days caused obvious enhancements in levels of Runx 2 (Figure 6C, top panel). Amounts of $\beta$-actin were quantified as the internal standard (Figure $6 \mathrm{C}$, bottom panel). These immunorelated protein bands were quantified and statistically analyzed (Figure 6D). After application on chitosan nanofibers for $1,3,5$, and 7 days, the levels of Runx 2 in human osteoblasts were significantly augmented by $86 \%$, $91 \%, 108 \%$, and $114 \%$, respectively. 


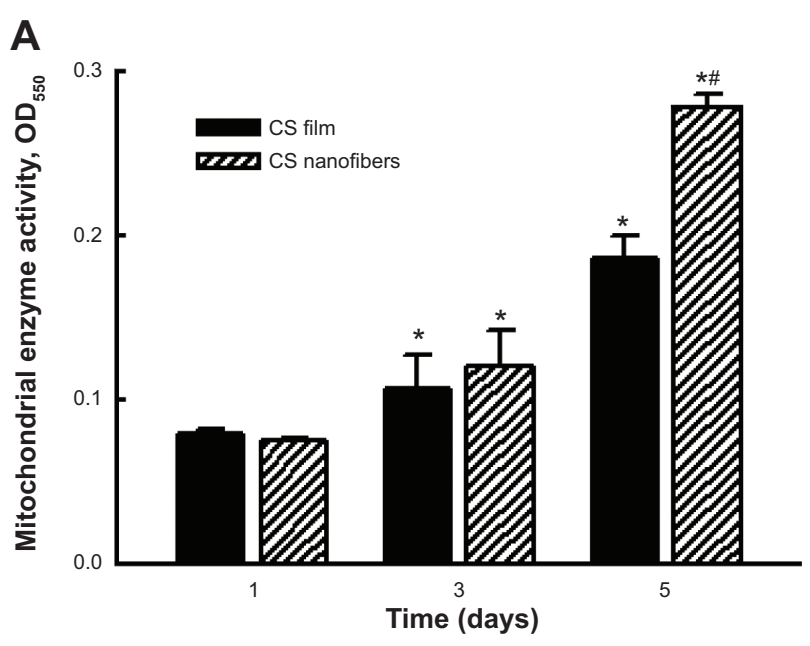

B
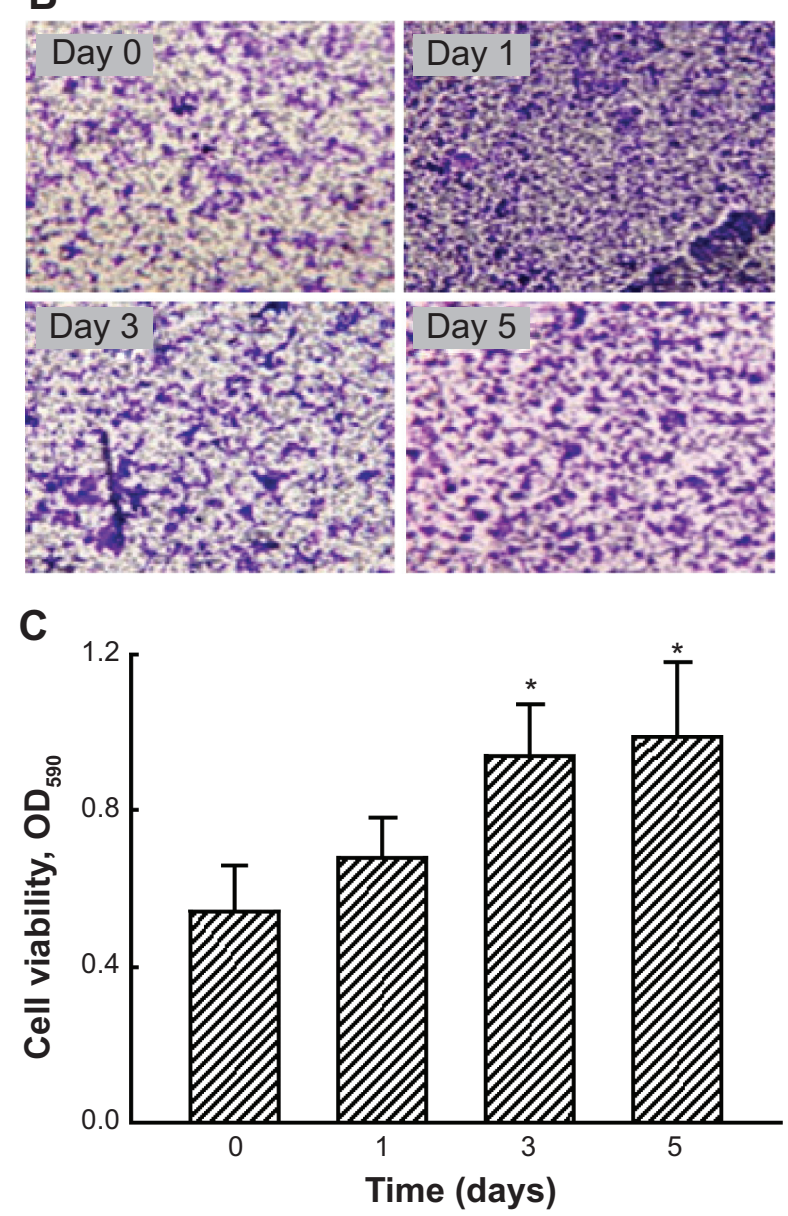

Figure 3 Effects of chitosan nanofibers on stimulating the growth of mouse osteoblasts.

Notes: Mouse osteosarcoma UMR-I06 cells were seeded on chitosan films and chitosan nanofiber scaffolds for I, 3, and 5 days. Cell growth was assayed by detecting mitochondrial complex I enzyme activity (A). Analysis of crystal violet staining was carried out to further verify the effects of chitosan nanofibers on the growth of mouse calvarial MC3T3-EI cells (B). Stained cells were dissolved, and the signals were quantified and statistically analyzed $(\mathbf{C})$. Each value represents the mean \pm standard error of the mean from four independent experiments. * and \# indicate that values significantly $(P<0.05)$ differed from the control and chitosan film-treated groups, respectively. $40 \times$ magnification.

Abbreviations: $\mathrm{CS}$, chitosan; $\mathrm{OD}_{550}$, optical density at $550 \mathrm{nM}$ wavelength; $\mathrm{OD}_{590}$, optical density at $590 \mathrm{nM}$ wavelength.

\section{Chitosan nanofiber scaffolds improved maturation of human osteoblasts}

Effects of the chitosan nanofiber scaffolds were determined using ALP-, alizarin red-, and von Kossa-staining protocols (Figure 7). Human osteoblasts were seeded on chitosan films and chitosan nanofiber scaffolds, and then exposed to a differentiation agent for 21 days. Compared to chitosan films, analysis by an ALP staining protocol revealed that chitosan nanofiber scaffolds increased mineralization of human osteoblasts by $92 \%$ (Figure 7A). In addition, analyses of alizarin red- and von Kossa-staining protocols further showed that seeding human osteoblasts on chitosan nanofiber scaffolds caused 3.8- and 2.5-fold increases in cell maturation, respectively (Figure 7B and C).

\section{Discussion}

This study provides evidence showing the potential of chitosan nanofiber scaffolds for bone tissue engineering. Bone tissues are maintained by bone remodeling. ${ }^{1}$ An imbalance in bone remodeling can delay the recovery and healing of bone defects and fractures, possibly leading to disability or even death. Presently, no effective drug can be clinically applied to treat bone defects and fractures. Fortunately, bone tissue engineering may be practical for therapy of bone diseases by making use of engineering and life sciences to enable bone tissue growth. ${ }^{16}$ Osteoblasts play a central role in mediating bone formation. ${ }^{2,3}$ Our present data revealed that chitosan nanofiber scaffolds stimulated growth of mouse osteosarcoma UMR-106 cells and mouse calvarial osteoblasts. Also, we further demonstrated that chitosan nanofibers significantly improved the growth and proliferation of human osteoblasts. Importantly, chitosan nanofibers were biocompatible when used for osteoblast culture. One major reason for orthopedic implant failure is the lack of sufficient integration of the implanted material with the juxtaposed bone. ${ }^{18}$ Chitosan nanofibers possess superior characteristics of interconnected micropores, optimal porosity, and absorption kinetics. Previous studies reported the applications of chitosan nanofibers for neurons, chondrocytes, and bone marrow mesenchymal stem cells. ${ }^{20,22-24}$ This study further shows the potential of chitosan nanofiber scaffolds for treating bone diseases such as bone defects and fractures.

The chitosan nanofiber scaffolds are biocompatible and provide an excellent space for osteoblast adhesion. The present study showed that, after being used with osteoblasts, the chitosan nanofibers degraded in a time-dependent manner. Biocompatibility is one of basic requirements for a qualified biomaterial. ${ }^{17}$ Chitosan is nontoxic, antibacterial, 
A

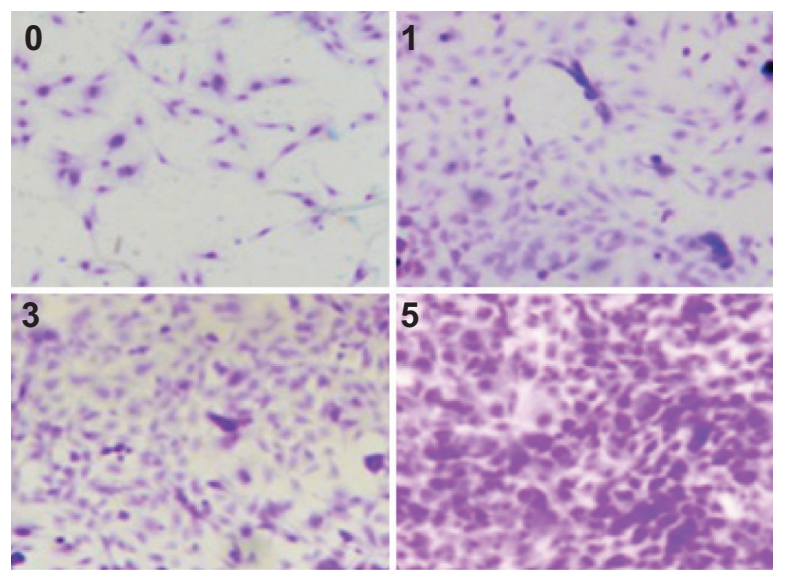

B

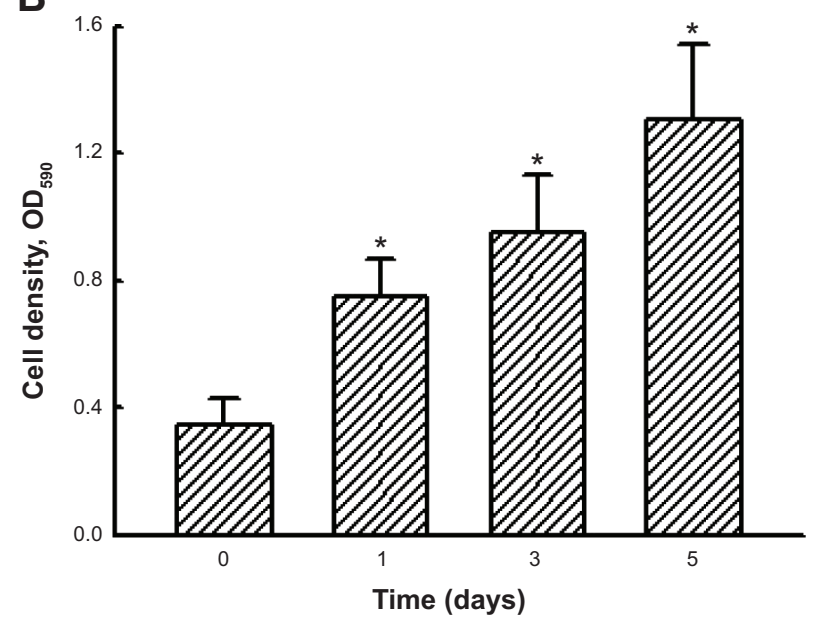

C

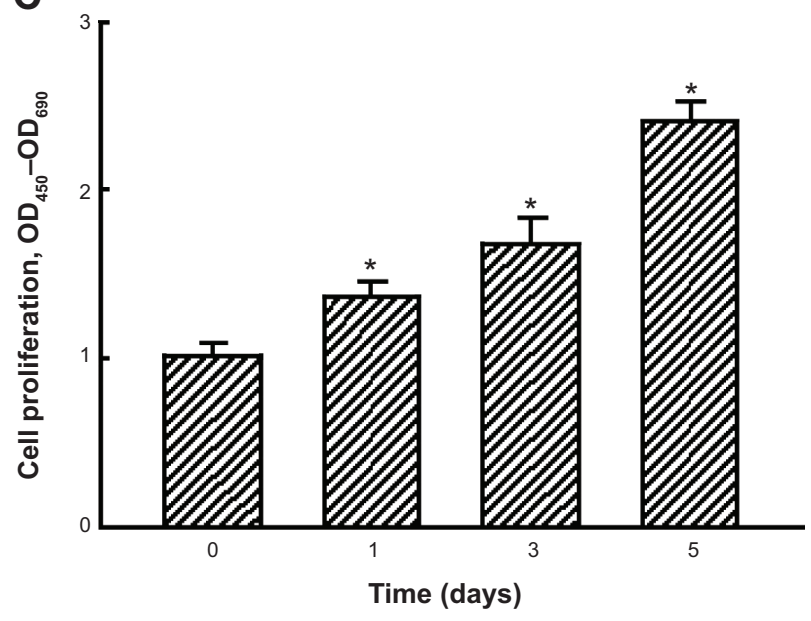

Figure 4 Effects of chitosan nanofibers on stimulating the growth and proliferation of human osteoblasts.

Notes: Human osteoblast-like MG63 cells were seeded on chitosan nanofiber scaffolds for I, 3, and 5 days. Growth of human osteoblasts was determined by crystal violet staining (A). Stained cells were dissolved, and the signals were quantified and statistically analyzed (B). Cell proliferation was assayed using an enzyme-linked immunosorbent assay bromodeoxyuridine kit (C). Each value represents the mean \pm standard error of the mean from four independent experiments. $*$ indicates that values significantly differed from the respective control, $P<0.05$. $40 \times$ magnification. Abbreviations: $\mathrm{OD}_{450}$, optical density at $450 \mathrm{nM}$ wavelength; $O D_{590}$, optical density at $590 \mathrm{nM}$ wavelength; $O D_{690}$, optical density at $690 \mathrm{nM}$ wavelength. and biocompatible. ${ }^{19}$ This study demonstrated that chitosan nanofibers did not change the morphologies or viabilities of mouse and human osteoblasts. Hence, chitosan nanofibers are biodegradable and nontoxic to osteoblasts. Cell adhesion is essential to maintaining a cell's activity and multicellular structure. ${ }^{33}$ Analyses by crystal violet staining and SEM showed that osteoblasts adhered onto chitosan nanofiber scaffolds and carried out cell-cell communication. A previous study reported that chitosan nanofibers/polycaprolactone scaffolds provided better bipolar elongation of neurons than nanofibrous substrates. ${ }^{20}$ Thus, chitosan nanofibers can stimulate adhesion of osteoblasts and, consequently, improve cell activity and cell maturation due to their higher surface areas and better porosities. Chitosan nanofibers possess these beneficial characteristics and displayed their novel application for bone tissue engineering.

Chitosan nanofibers can stimulate cell proliferation and growth. Our present data reveal that mouse osteoblasts grew better on chitosan nanofibers than on chitosan films. In addition to mouse osteosarcoma cells, our findings also showed improved effects of chitosan nanofibers on the growth of mouse calvarial osteoblasts and human osteoblasts. Prabhakaran et al stated that electrospun nanofibers were able to provide greater porosity for cell adhesion. ${ }^{20}$ Thus, the advantageous effects of enhancing cell adhesion may explain the chitosan nanofiber-induced stimulation of osteoblast growth. Our BrdU incorporation assay further showed that after being seeded onto chitosan nanofiber scaffolds, DNA replication of human osteoblasts was significantly enhanced. Augmentation of DNA replication corresponds to an increase in cell proliferation. ${ }^{34}$ This study demonstrated the effects of the chitosan nanofibers on inducing OPN gene expression. OPN can regulate cell proliferation. ${ }^{11}$ Hence, chitosan nanofibers can enhance the proliferation of human osteoblasts via stimulating DNA replication due to the induction of OPN gene expression. Borjigin et al reported that electrospun fibers can facilitate the proliferation of genetically modified HCT116-19 colon cancer cells. ${ }^{35}$ In the progression of bone development, the proliferation of osteoprogenitors and osteoblasts are key stages. ${ }^{5,6}$ This study pointed out that chitosan nanofibers can improve bone development by stimulating OPN-mediated osteoblast proliferation and growth.

Chitosan nanofibers can induce osteoblast differentiationrelated gene expression and promote osteoblast maturation. Analysis by ALP staining showed that, after being cultured on chitosan nanofiber scaffolds, the amount of osteoblasts with positive signals significantly increased. ALP is a biomarker of the regulation of osteoblast activity 
A

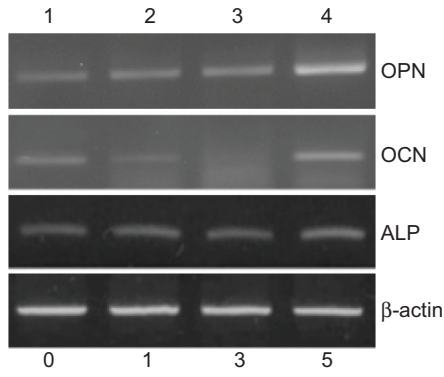

C

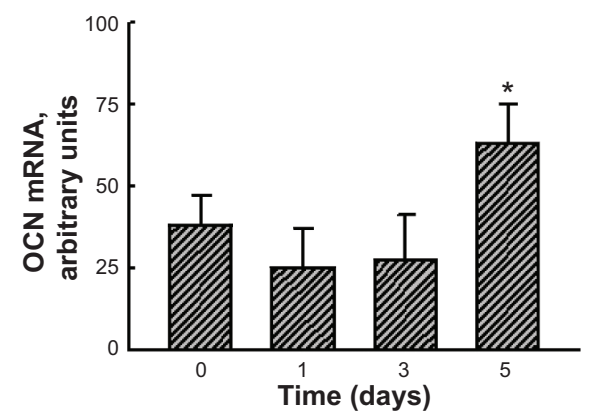

B
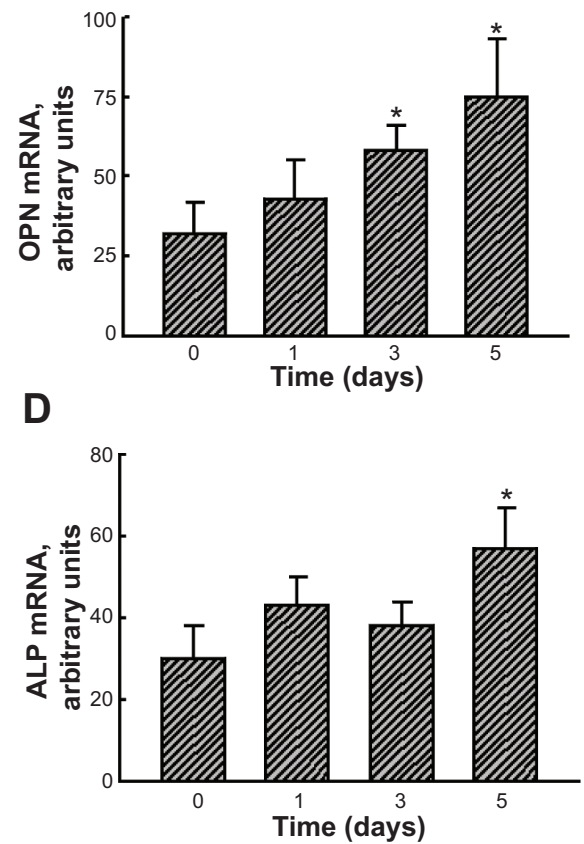

Figure 5 Effects of chitosan nanofibers on the induction of osteoblast differentiation-related OPN, OCN, and ALP gene expression.

Notes: Human osteoblast-like MG63 cells were seeded on chitosan nanofiber scaffolds for I, 3, and 5 days. (A) Analyses of OPN, OCN, and ALP mRNAs were conducted using RT-PCR; $\beta$-actin mRNA was analyzed as an internal control. The DNA bands were quantified and statistically analyzed (B-D). Each value represents the mean \pm standard error of the mean from four independent experiments. * indicates that values significantly differed from the respective control, $P<0.05$.

Abbreviations: ALP, alkaline phosphatase; mRNA, messenger RNA; OCN, osteocalcin; OPN, osteopontin; RT-PCR, reverse transcription polymerase chain reaction.

A

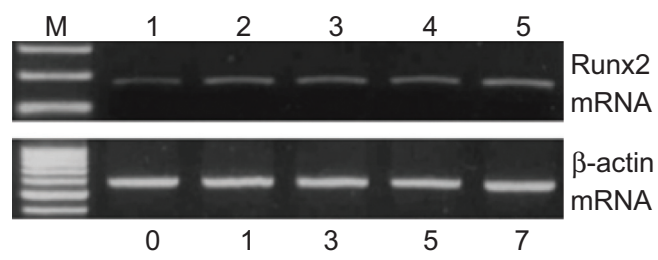

C

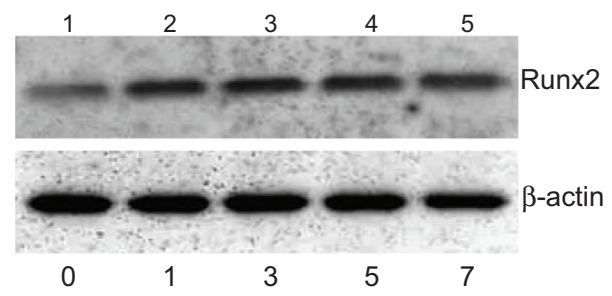

B
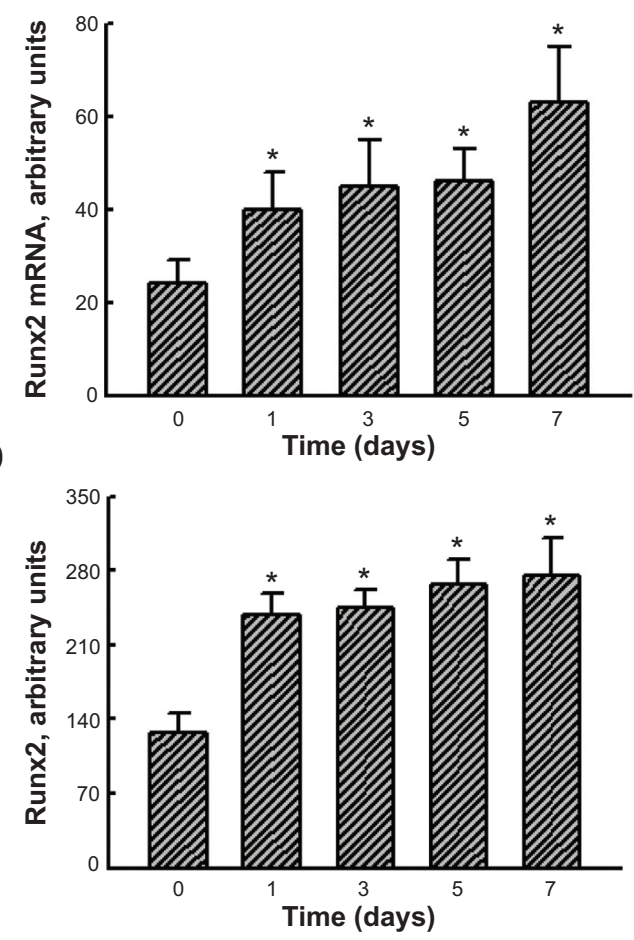

Figure 6 Effects of chitosan nanofibers on the induction of Runx2 mRNA and protein expressions

Notes: Human osteoblast-like MG63 cells were seeded on chitosan nanofiber scaffolds for I, 3, 5, and 7 days. (A) Runx2 mRNA was analyzed using RT-PCR; $\beta$-actin mRNA was analyzed as an internal control. The DNA bands were quantified and statistically analyzed (B). (C) Runx2 was detected using a mouse monoclonal antibody; $\beta$-actin was immunodetected as an internal control. The immunoreactive protein bands were quantified and statistically analyzed (D). Each value represents the mean \pm standard error of the mean from four independent experiments. ${ }^{*}$ indicates that values significantly differed from the respective control, $P<0.05$.

Abbreviations: mRNA, messenger RNA; Runx2, runt-related transcription factor 2; RT-PCR, reverse transcription polymerase chain reaction. 

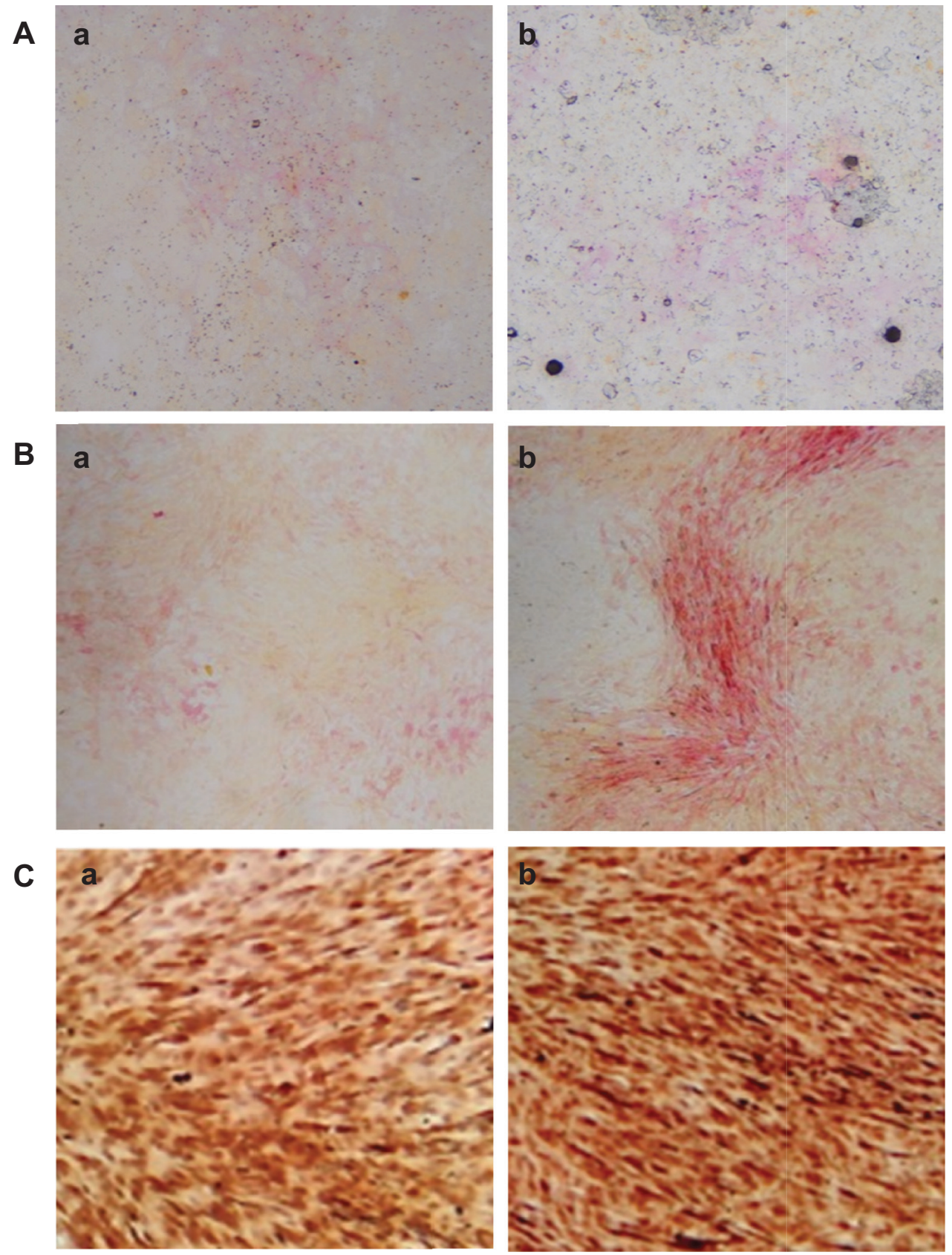

Figure 7 Effects of chitosan nanofibers on the mineralization of human osteoblasts.

Notes: Human osteoblast-like MG63 cells were seeded on chitosan films and chitosan nanofiber scaffolds, and then exposed to a differentiation reagent ( $10 \mathrm{nM}$ dexamethasone, $100 \mu \mathrm{g} / \mathrm{mL}$ ascorbic acid, and $10 \mathrm{mM} \beta$-glycerophosphate) for 21 days. The differentiation reagent was renewed every 2 days. Mineralization of human osteoblasts was determined using ALP-, Alizarin red S dye-, and von Kossa-staining protocols. (A) ALP staining: (a) chitosan film and (b) chitosan nanofibers. (B) Alizarin red staining: (a) chitosan film and (b) chitosan nanofibers. (C) von-Kossa staining: (a) chitosan film and (b) chitosan nanofibers. 40× magnification.

Abbreviation: ALP, alkaline phosphatase.

and maturation. ${ }^{3,9}$ An increase in ALP staining signals correspondingly indicates enhancement of osteoblast maturation. Such an effect of chitosan nanofibers on osteoblast development was also demonstrated by the Alizarin red- and von Kossa-staining assays. A complicated array of molecular events participates in the regulation of osteogenesis and bone maturation. ${ }^{7,8}$ In addition, a variety of osteoblast-associated genes tightly control these progressive molecular and cellular events. ${ }^{7,16}$ The present study showed the effects of chitosan nanofibers on inducing OPN, OCN, and ALP gene expression. During osteoblast differentiation, these three marker genes can be regulated individually.7,9 Our previous study has demonstrated that exposure of osteoblasts to the water extracts of Drynaria fortunei J. Smith, a traditional 
Chinese medicine, for 3 days could induce OPN, OCN, and ALP gene expression. ${ }^{36}$ In the present study, OPN mRNA was induced by chitosan nanofibers earlier than the other two marker genes. OPN, OCN, and ALP are all early osteoblast markers that control osteoblast differentiation and bone ECM mineralization. ${ }^{10}$ In addition to osteoprogenitor proliferation, matrix maturation and cell mineralization are two final stages in the process of osteoblast development. ${ }^{5,6}$ Therefore, chitosan nanofiber-induced osteoblast maturation may have been due to regulation of these osteoblast differentiation-related gene expression.

Runx2 participates in chitosan nanofiber-induced osteoblast mineralization. Exposure of human osteoblasts to chitosan nanofibers caused a significant induction of Runx2 mRNA expression. In parallel, the synthesis of Runx 2 protein was augmented following exposure to chitosan nanofibers. Thus, chitosan nanofiber-induced Runx 2 expression occurs at least at a pretranslational level. During osteogenic differentiation, bone morphogenetic proteins (BMPs) were shown to activate Runx $2 .{ }^{37}$ Our previous study showed that BMP-2 and -6 were detected and induced in osteoblasts. ${ }^{36}$ Chitosan nanofibers may induce Runx 2 gene expression via the BMP signaling pathway. Runx2 functions as an osteogenic master transcription factor and regulates osteoblast differentiation and maturation. ${ }^{12,13}$ The p300/CBP-associated factor is reported to activate Runx 2 by directly binding to and acetylating this transcription factor. ${ }^{14}$ Knockdown of p300/ CBP-associated factor gene expression simultaneously leads to Runx 2 inactivation and subsequent downregulation of OPN synthesis. Runx 2 was also demonstrated to transcriptionally regulate OCN and ALP gene expression. ${ }^{38}$ Therefore, chitosan nanofibers induced OPN, OCN, and ALP gene expression via a Runx2-dependent mechanism and, consequently, improved osteoblast differentiation and mineralization.

\section{Conclusion}

In conclusion, we developed chitosan nanofibers that promoted adhesion of mouse osteoblasts. Analysis by SEM further showed the attachment of mouse osteoblasts onto electrospun scaffolds along the nanofibers and formation of cell-cell communication. Mouse osteoblasts grew much better on chitosan nanofiber scaffolds compared to chitosan films. In addition, the present study demonstrated that human osteoblasts can adhere to and grow on chitosan nanofiber scaffolds. Interestingly, applying human osteoblasts to chitosan nanofibers stimulated DNA replication and cell proliferation. In parallel, expression of OPN, OCN, and ALP genes by human osteoblasts were induced after exposure to chitosan nanofiber scaffolds. Sequentially, chitosan nanofibers augmented Runx 2 mRNA and protein syntheses. Consequently, analyses using ALP-, Alizarin red-, and von Kossa-staining protocols further showed that chitosan nanofibers improved mineralization of human osteoblasts. Taken together, this study showed that chitosan nanofibers can stimulate osteoblast proliferation and maturation via Runx2-mediated regulation of osteoblast-associated OPN, OCN, and ALP gene expression. Therefore, chitosan nanofibers have the potential to be clinically applied to treat bone diseases such as bone defects and fractures.

\section{Acknowledgments}

This study was supported by Taipei Medical University and National Taiwan University of Science and Technology (TMU-NTUST-100-03), Wan-Fang Hospital (100-wfeva-17), and the Ministry of Science and Technology (NSC101-2314-B-038-003-MY3), Taipei, Taiwan.

\section{Disclosure}

The authors report no conflicts of interest in this work.

\section{References}

1. Seeman E, Delmas PD. Bone quality - the material and structural basis of bone strength and fragility. New Engl J Med. 2006;354:2250-2261.

2. Ducy P, Schinke T, Karsenty G. The osteoblast: a sophisticated fibroblast under central surveillance. Science. 2000;289:1501-1504.

3. Chen RM, Lin YL, Chou CW. GATA-3 transduces survival signals in osteoblasts through upregulation of $b c l-x L$ gene expression. J Bone Min Res. 2010;25:2193-2204.

4. Aubin JE, Liu F, Malaval L, Gupta AK. Osteoblast and chondroblast differentiation. Bone. 1995;17:77S-83S.

5. Giustina A, Mazziotti G, Canalis E. Growth hormone, insulin-like growth factors, and the skeleton. Endocrine Rev. 2008;29:535-559.

6. Aubin JE. Bone stem cells. J Cell Biochem. 1998;30-31:73-82.

7. Stein GS, Lian JB, Stein JL, Van Wijnen AJ, Montecino M. Transcriptional control of osteoblast growth and differentiation. Physiol Rev. 1996;76:593-624.

8. Vandenput L, Ohlsson C. Estrogens as regulators of bone health in men. Nature Rev Endocrinol. 2009;5:437-443.

9. Zhou H, Choong P, McCarthy R, Chou ST, Martin TJ, Ng KW. In situ hybridization to show sequential expression of osteoblast gene markers during bone formation in vivo. J Bone Miner Res. 1994;9:1489-1499.

10. van Leeuwen JP, van Driel M, van den Bemd GJ, Pols HA. Vitamin D control of osteoblast function and bone extracellular matrix mineralization. Crit Rev Eukaryot Gene Expr. 2001;11:199-226.

11. Haylock DN, Nilsson SK. Osteopontin: a bridge between bone and blood. Br J Haematol. 2006;134:467-474.

12. Hawse JR, Subramaniam M, Ingle JN, Oursler MJ, Rajamannan NM, Spelsberg TC. Estrogen-TGF-b cross-talk in bone and other cell types: role of TIEG, Runx2, and other transcription factors. $J$ Cell Biochem. 2008;103:383-392.

13. Ho WP, Chan WP, Hsieh MS, Chen RM. Runx2-mediated bcl-2 gene expression contributes to nitric oxide protection against oxidative stressinduced osteoblast apoptosis. J Cell Biochem. 2009;108:1084-1093.

14. Inman CK, Shore P. The osteoblast transcription factor Runx2 is expressed in mammary epithelial cells and mediates osteopontin expression. J Biol Chem. 2003;278:48684-48689. 
15. Wang CY, Yang SF, Wang Z, et al. PCAF acetylates Runx 2 and promotes osteoblast differentiation. J Bone Miner Metab. 2013;31:381-389.

16. Huang Z, Tian J, Yu B, Xu Y, Feng Q. A bone-like nano-hydroxyapatite/ collagen loaded injectable scaffold. Biomed Mater. 2009;4:055005.

17. Serrano F, Lopez L, Jadraque M, et al. YAG Laser-microperforated poly(3-hydroxybutyrate-co-3-hydroxyvalerate)-basal membrane matrix composite film as substrate for keratinocytes. Biomaterials. 2007;28:650-660.

18. Divya Rani VV, Manzoor K, Menon D, Selvamurugan N, Nair SV. The design of novel nanostructures on titanium by solution chemistry for an improved osteoblast response. Nanotechnology. 2009;20:195101.

19. Jayakumar R, Prabaharan M, Nair SV, Tamura H. Novel chitin and chitosan nanofibers in biomedical applications. Biotechnol Adv. 2010;28:142-150.

20. Prabhakaran MP, Venugopal JR, Chyan TT, et al. Electrospun biocomposite nanofibrous scaffolds for neural tissue engineering. Tissue Eng Part A. 2008;14:1787-1797.

21. Wang W, Itoh S, Konno K, et al. Effects of Schwann cell alignment along the oriented electrospun chitosan nanofibers on nerve regeneration. J Biomed Mater Res Part A. 2009;91:994-1005.

22. Chen ZG, Wang PW, Wei B, Mo XM, Cui FZ. Electrospun collagenchitosan nanofiber: a biomimetic extracellular matrix for endothelial cell and smooth muscle cell. Acta Biomate. 2010;6:372-382.

23. Jancár J, Slovíková A, Amler E, et al. Mechanical response of porous scaffolds for cartilage engineering. Physiol Res. 2007;56 Suppl 1: S17-S25.

24. Liu H, Peng H, Wu Y, et al. The promotion of bone regeneration by nanofibrous hydroxyapatite/chitosan scaffolds by effects on integrinBMP/Smad signaling pathway in BMSCs. Biomaterials. 2013;34: 4404-4417.

25. Desai K, Kit K, Li J, Zivanovic S. Morphological and surface properties of electrospun chitosan nanofibers. Biomacromolecules. 2008;9: $1000-1006$.

26. Liao MH, Tai YT, Cherng YG, et al. Genistein induces estrogen receptor- $\alpha$ gene expression in osteoblasts through activation of MAPKs/ NF-KB/AP-1 and promotes cell mineralization. Br J Nutr. 2014;111: $55-63$.
27. Kueng W, Silber E, Eppenberger U. Quantification of cells cultured on 96-well plates. Anal Biochem. 1989;182:16-19.

28. Mogi M, Togari A. Activation of caspases is required for osteoblastic differentiation. J Biol Chem. 2003;278:47477-47482.

29. Wu TT, Tai YT, Cherng YG, et al. GATA-2 transduces LPS-induced il-1 $\beta$ gene expression in macrophages via a toll-like receptor 4/MD88/ MAPK-dependent mechanism. PloS One. 2013;8:e72404.

30. Zhao Y, Guan H, Liu SF, Wu RC, Wang Z. Overexpression of QM induces cell differentiation and mineralization in MC3T3-E1. Biol Pharm Bull. 2005;28:1371-1376.

31. Chen RM, Tai YT, Chen TG, et al. Propofol protects against nitrosative stress-induced breakage of the blood-brain barrier through reducing apoptotic insults to cerebrovascular endothelial cells. Surgery. 2013;154:58-68.

32. Alcantara EH, Shin MY, Sohn HY, et al. Diosgenin stimulates osteogenic activity by increasing bone matrix protein synthesis and bonespecific transcription factor Runx2 in osteoblastic MC3T3-E1 cells. J Nutr Biochem. 2011;22:1055-1063.

33. Liddington RC, Ginsberg MH. Integrin activation takes shape. $J$ Cell Biol. 2002;158:833-839.

34. Raouf A, Seth A. Ets transcription factors and targets in osteogenesis. Oncogene. 2000;19:6455-6463.

35. Borjigin M, Eskridge C, Niamat R, Strouse B, Bialk P, Kmiec EB. Electrospun fiber membranes enable proliferation of genetically modified cells. Int J Nanomedicine. 2013;8:855-864.

36. Huang TY, Chen TL, Liao MH, et al. Drynaria fortunei J. Sm. promotes osteoblast maturation by inducing differentiation-related gene expression and protecting against oxidative stress-induced apoptotic insults. J Ethnopharmacol. 2010;131:70-77.

37. Trzeciakiewicz A, Habauzit V, Mercier S, et al. Hesperetin stimulates differentiation of primary rat osteoblasts involving the BMP signalling pathway. J Nutr Biochem. 2010;21:424-431.

38. Wang H, Huo N, Li F, et al. Osteogenic role of endosomal chloride channels in MC3T3-E1 cells. Mol Cell Biochem. 2010;342:191-199.
International Journal of Nanomedicine

\section{Publish your work in this journal}

The International Journal of Nanomedicine is an international, peerreviewed journal focusing on the application of nanotechnology in diagnostics, therapeutics, and drug delivery systems throughout the biomedical field. This journal is indexed on PubMed Central, MedLine, CAS, SciSearch ${ }^{\circledR}$, Current Contents ${ }^{\circledR} /$ Clinical Medicine,

\section{Dovepress}

Journal Citation Reports/Science Edition, EMBase, Scopus and the Elsevier Bibliographic databases. The manuscript management system is completely online and includes a very quick and fair peer-review system, which is all easy to use. Visit http://www.dovepress.com/ testimonials.php to read real quotes from published authors. 